\title{
Neutron beam test with 3D-projection scintillator tracker prototypes for long-baseline neutrino oscillation experiments
}

\author{
Guang Yang ${ }^{a, *}$ \\ ${ }^{a}$ Stony Brook University, \\ 100 Nicolls Road, Stony Brook, NY, USA \\ E-mail: guang.yang.1@stonybrook.edu
}

The long-baseline neutrino oscillation experiments rely on detailed models of neutrino interactions on nuclei to relate observations made at near detector locations to far detector locations in order to extract precision measurements on the oscillation parameters. These models constitute an important source of systematic uncertainty, driven in part because detectors to date have been blind to the neutrons produced in neutrino interactions. We are proposing a 3D-projection scintillator tracker as a near detector component in the next generation long-baseline neutrino experiments such as T2K upgrade and DUNE. Such a detector consists of a large number of 1 $\mathrm{cm} \times 1 \mathrm{~cm} \times 1 \mathrm{~cm}$ scintillator cubes with three orthogonal optical fibers crossing through each cube. Benefitted by the good timing resolution and fine granularity, this detector will be capable of measuring neutrons including its kinetic energy in neutrino interactions on an event-by-event basis and thus will provide valuable data for refining neutrino interaction models. Two prototypes have been exposed to the neutron beamline in Los Alamos National Lab (LANL) with neutron energy ranged from 0 to $800 \mathrm{MeV}$. This beam test, aimed at characterizing the detectors' response to neutrons, is a critical step in demonstrating the potential of this technology. In this paper, the prototype detectors and the LANL beam test setup will be described, and the preliminary event displays will be shown.

40th International Conference on High Energy physics - ICHEP2020

July 28 - August 6, 2020

Prague, Czech Republic (virtual meeting)

\footnotetext{
${ }^{*}$ Speaker
} 


\section{Introduction}

The future long-baseline neutrino oscillation experiments accomplish a measurement of the $\mathrm{CP}$ violating phase with an unprecedented precision by aiming beams of neutrinos and anti-neutrinos at a detector located several hundred kilometers or more away and measuring the oscillation of the (anti)neutrinos between flavor eigenstates. In order to discern the oscillation phenomena, the experiments reconstruct the energy of the neutrino or anti-neutrino that undergo charged current interactions in the detector based on the resultant visible particles. While it is relatively straightforward to reconstruct charged particles, neutrons present a special challenge. Accounting for the energy in the form of neutrons typically results in large systematic uncertainties. Given common challenges, the DUNE and T2K experimentshave embarked on a joint R\&D program to develop a high-quality plastic scintillator-based near detector. The fine-grained tracking detector design is composed of optically isolated plastic scintillator cubes with a size of $1 \mathrm{~cm} \times 1 \mathrm{~cm} \times 1 \mathrm{~cm}$. Three wavelength shifting fibers cross each cube in three dimensions and a readout MPPC is attached on one end of the fiber [1]. Such a scintillator tracker, also called 3DST, provides three 2D views and a combination of those views can form a 3D reconstructed image. There are three key features of this tracker : fine-grained spatial resolution ( $<3 \mathrm{~mm}$ space point resolution in $2 \mathrm{D}$ space), fast timing ( 1 ns resolution for a single channel with light from a minimum ionizing particle [2]) and excellent neutron sensitivity (high efficiency $10 \mathrm{MeV}$ and above [3]).

\section{Detector and experiment setup}

Two 3DST prototypes have been assembled and delivered to Los Alamos National Lab Weapon Nucluear Research (WNR) facility. One has a dimension of $24 \mathrm{~cm} \times 8 \mathrm{~cm} \times 48 \mathrm{~cm}$ (superFGD prototype) while the other $8 \mathrm{~cm} \times 8 \mathrm{~cm} \times 32 \mathrm{~cm}$ (US-Japan prototype). Those two prototype detectors were proposed and built by a collaboration with scientists from 14 institutions in 6 countries and CERN. The superFGD prototype has been exposed to a charged particle beamline in CERN and the results can be found in [4]. The WNR facility provides a neutron beam with energy ranged from 0 to $800 \mathrm{MeV}$. Each beam bundle is separated by $1.8 \mu \mathrm{s}$. Two separate beamline time allocations at $90 \mathrm{~m}$ and $20 \mathrm{~m}$ locations from the proton target were approved. A gamma flash, which provides a t0 signal, comes before the neutron arrives, which allows a neutron energy measurement with the time-of-flight. Given the outstanding time resolution in the detector, time difference between the gamma flash and the neutron arrival time has been used to obtain a $2 \%$ level energy resolution across a broad energy range. Fig. 1 shows the principle of the time-of-flight technique and what we observed in the detector.

\section{Physics measurements and plans}

The prototype detectors can measure each individual neutron interaction in the beamline and each individual interaction is analyzed based on the topology, time and energy deposit information. Fig. 2 shows two example neutron-induced event candidates. The beam is from the right to the left and the color corresponds to the detected charges. As a consequence, a number of neutron interaction deliverables will be extracted and input to the neutrino interaction modeling. The useful 


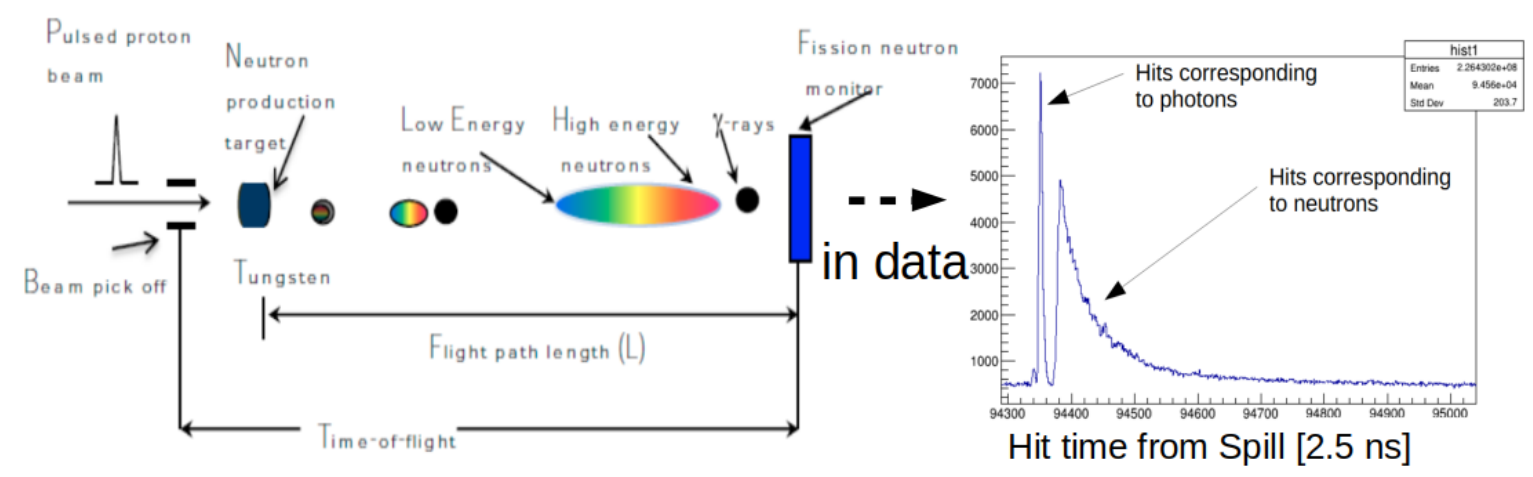

Figure 1: The principle of measuring neutron energy with time-of-flight and the time distribution measured in the detector.

information include the detection efficiency as function of the neutron energy, neutron-induced signal topology, neutron double-scattering rate and topology etc. As a first step, a total neutron cross section measurement as a function of neutron energy has being pursued using the so-called extinction method. With such a method, at each energy bin, an exponential curve is fit to the neutron interaction vertices along the beam direction. The exponential coefficient can provide a direct result of the neutron cross section. This analysis is expected to be done in a few months time scale. In addition, a second round beam test is being done in Dec. 2020 at WNR with more flexible beam conditions.
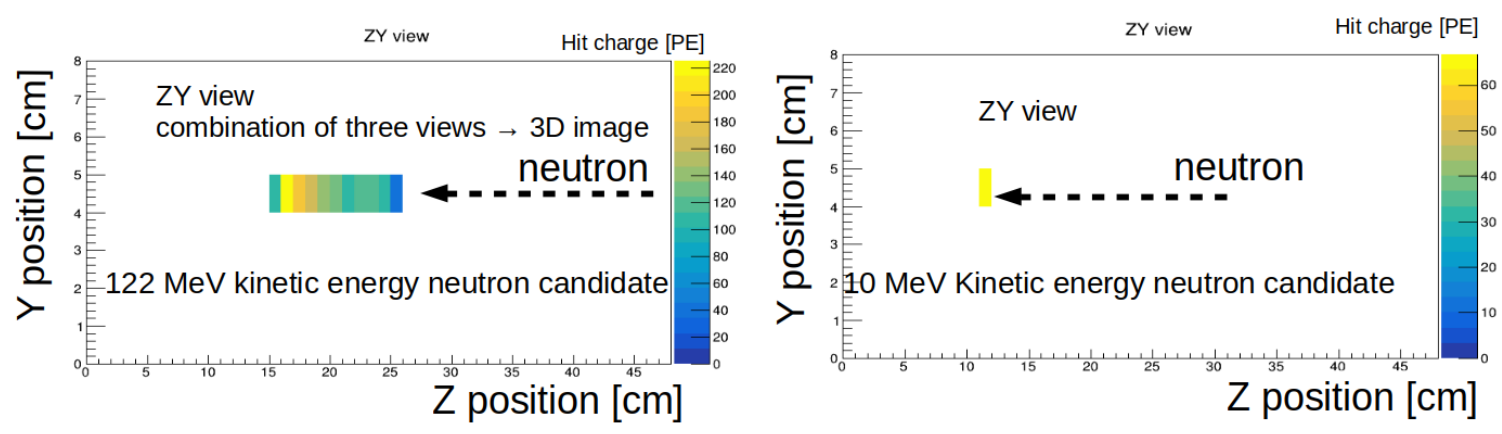

Figure 2: Two neutron-induced candidates on one of the three views in the superFGD prototype.

\section{References}

[1] A. Blondel et al., Journal of Instrumentation, 13, P02006 (2018).

[2] O. Mineev et al., 10.1016/j.nima.2019.01.080

[3] L. Munteanu et al., Phys. Rev. D 101, 092003

[4] A. Blondel et al., arXiv:2008.08861[physics.ins-det]. 